\title{
Endoscopy evaluation to predict oral appliance outcomes in obstructive sleep apnoea
}

\author{
Kentaro Okuno ${ }^{1,2}$, Yasuhiro Sasao ${ }^{2,3}$, Kanji Nohara2, Takayoshi Sakai², \\ Benjamin T. Pliska ${ }^{1}$, Alan A. Lowe ${ }^{1}$, C. Frank Ryan ${ }^{4}$ and Fernanda R. Almeida ${ }^{1}$
}

Affiliations: ${ }^{1}$ Dept of Oral Health Sciences, Faculty of Dentistry, University of British Columbia, Vancouver, BC V6T 1Z3, Canada. ${ }^{2}$ Division of Functional Oral Neuroscience, Osaka University Graduate School of Dentistry, Osaka 565-0871, Japan. ${ }^{3}$ Center of Oral Functional Disorders, Sasao Dental Clinic, Yamaguchi Prefecture 7400027, Japan. ${ }^{4}$ Division of Respiratory Medicine, Dept of Medicine, University of British Columbia, Vancouver, BC V6T 123, Canada.

Correspondence: Kentaro Okuno, Dept of Oral Health Sciences, Faculty of Dentistry, University of British Columbia, 2199 Wesbrook Mall, Vancouver, BC V6T 1Z3, Canada. E-mail: k-okunoddent.osaka-u.ac.jp

ABSTRACT The objective of this study was to determine the utility of nasoendoscopy of the upper airway as a predictor of the efficacy of oral appliance treatment in obstructive sleep apnoea (OSA).

A total of 61 consecutive patients with moderate-to-severe polysomnographically diagnosed OSA were recruited for this study. Using nasoendoscopy, we prospectively assessed the velopharynx and oro/ hypopharynx in each patient while awake and in the supine position. We measured cross-sectional area (CSA), and anteroposterior and lateral diameters of the airway before and after mandibular advancement, and expressed the changes in dimensions as expansion ratios (after/before). We then compared the measurements of responders and nonresponders with oral appliance treatment.

The expansion ratio (median (interquartile range)) for the CSA was greater in responders compared with nonresponders in the velopharynx (2.9 (2.3-5.0) versus $1.7(1.5-1.9), \mathrm{p}<0.001)$ and in the oro/ hypopharynx $(3.4(2.5-5.6)$ versus $2.4(1.8-3.7), \mathrm{p}<0.05)$. Baseline apnoea-hypopnoea index and the CSA expansion ratio of the velopharynx were independent predictors of oral appliance treatment outcome based on a multivariate logistic regression analysis. The estimated area under the receiver operator characteristic curve was 0.87 and the cut-off value of the expansion ratio was 2.00 .

These results indicate that nasoendoscopy may have significant clinical utility in predicting the success of oral appliance treatment.

@ERSpublications

A pronounced increase of the velopharynx with mandibular protrusion is a good predictor of oral appliance success http://ow.ly/V9lBU 


\section{Introduction}

Obstructive sleep apnoea (OSA) is a common syndrome that is associated with reduced quality of life, decreased cardiovascular health, and increased healthcare utilisation, motor vehicle accidents and mortality $[1,2]$. Treatment with an oral appliance is an alternative to continuous positive airway pressure (CPAP), although it appears to be less efficacious but more accepted by patients [3-7]. An American Academy of Sleep Medicine and American Academy of Dental Sleep Medicine clinical practice guideline recommends an oral appliance as a treatment for adult patients with OSA who either prefer oral appliance therapy or are intolerant of CPAP therapy [8]. A recent review reported that oral appliances are an efficacious treatment option for OSA in $60-70 \%$ of patients [9], and demonstrated that oral appliances can improve sleep-disordered breathing and daytime symptoms in a substantial number of patients with the disease. Hence, the ability to predict the efficacy of oral appliance treatment in individual patients, especially those with moderate to severe OSA, would be highly desirable for efficient clinical care [10].

Nasoendoscopy has been reported previously as a useful approach to assessing the upper airway and may be able to predict response to soft tissue surgical treatment of OSA [11]. In terms of oral appliance treatment success, Johal et al. $[12,13]$ and BATTAGel et al. [14] reported that sleep nasoendoscopy could be a valuable prognostic tool of successful oral appliance treatment outcome, and VROEGOP et al. [15] showed a significant association between the findings during drug-induced sleep endoscopy (DISE) and treatment response with oral appliance. Endoscopic evaluation during wakefulness has also been reported to be useful for predicting oral appliance treatment response $[16,17]$. However, methodological limitations of these previous studies, such as the qualitative assessments and retrospective analysis of the data, have restricted their clinical usefulness.

In the current study, we prospectively and quantitatively evaluated the morphological changes of the upper airway with mandibular protrusion using nasoendoscopy to predict oral appliance treatment outcome. The aim of the present study was to better define and simplify previous nasoendoscopy techniques by the use of more exact measurements of pharyngeal dimensions with and without mandibular advancement in awake patients in the supine position.

\section{Methods \\ Subjects}

Subjects were prospectively recruited for study participation from patients referred to the Osaka University Dental Hospital between 2007 and 2012 for oral appliance treatment of sleep-disordered breathing. Patients with moderate (apnoea-hypopnoea index (AHI) $\geqslant 15$ and $<30$ events $\cdot \mathrm{h}^{-1}$ ) and severe (AHI $\geqslant 30$ events $\cdot \mathrm{h}^{-1}$ ) OSA diagnosed by overnight attended polysomnography (PSG) using standard parameters [18, 19] were selected for this study. Patients were excluded if they had severe periodontal disease, insufficient number of teeth, temporomandibular joint dysfunction, prior otolaryngeal surgery, cardiovascular disease, neuromuscular disease or were medically unstable. Baseline characteristics of the patients are shown in table 1. A total of 61 patients consented to participation and met the defined eligibility criteria to be enrolled in the study. This study was approved by the Ethics Committee of Osaka University Dental Hospital (H19-E3-1) and written informed consent was obtained from all patients.

\section{Polysomnography}

PSG was performed to confirm the OSA diagnosis and to determine treatment outcome according to standard criteria $[18,19]$. Standard measurements included electroencephalography, electrooculography, submental electromyography, electrocardiography, chest and abdominal respiratory impedance plethysmography, arterial oxygen saturation by pulse oximetry $\left(\mathrm{SpO}_{2}\right)$, and nasal airflow (nasal cannulae connected to a pressure transducer) were recorded. Apnoea was defined as the cessation of airflow at

\section{TABLE 1 Patient characteristics at baseline}

$\begin{array}{lc}\text { Subjects } \mathbf{n} & 61 \\ \text { Male } \% & 82.0 \\ \text { Age years } & 56.0 \pm 10.9 \\ \text { BMI kg:m }{ }^{-2} & 26.2 \pm 3.7 \\ \text { AHI baseline events } \cdot \mathbf{h}^{-1} & 35.8 \pm 17.0 \\ \text { ESS baseline } & 10.6 \pm 4.6\end{array}$

Data are presented as mean $\pm \mathrm{SD}$, unless otherwise stated. BMI: body mass index; AHI: apnoea-hypopnoea index; ESS: Epworth sleepiness scale. 
$\geqslant 10 \mathrm{~s}$. Hypopnoea was defined as a respiratory event that caused a $\geqslant 50 \%$ reduction in airflow for $\geqslant 10 \mathrm{~s}$ associated with $a \geqslant 3 \%$ peripheral artery oxygen desaturation $\left(\mathrm{SpO}_{2}\right)$ or resulted in an arousal.

\section{Endoscopy and test task}

Endoscopy was performed using a flexible nasoendoscope (FNL-10RP3; Pentax, Tokyo, Japan) by the same clinician with $>7$ years clinical experience in this technique. Following the administration of topical anaesthetic nasal spray (4\% lignocaine) to one nasal passage, the nasoendoscope was inserted with the patient in the supine position and the Frankfort plane perpendicular to the floor. In order to maintain the same head and neck position during measurements, the subject's head was supported by the headrest of dental chair. The clinician ensured a constant head position with visual monitoring during measurements. With the endoscope positioned at standardised positions, first at the level of the velopharynx (the soft palate and the uvula) and then at the oro/hypopharynx (between the uvula and the epiglottis), each patient was studied while awake during quiet nasal breathing with the mouth closed and asked to protrude the mandible while keeping contact between the upper and lower anterior teeth, from the centric occlusal position (habitual occlusion with maximum intercuspation of the teeth) to the maximum comfortable protrusion, as previously described [16]. In order to maintain a consistent distance during measurements, the fibrescope was fixed on the nares with tape. Prior to image capture, subjects were given instructions for mandibular advancement and given time to practice until they were stable performing it. Static digital images of the velopharynx and oro/hypopharynx at these two mandibular positions were captured by video-editing software at the end-tidal expiration as determined by visual monitoring on the display and chest movement, as previously described [20]. This display was only visible by the clinician.

\section{Evaluation methods}

Assessment of nasoendoscopic findings of the upper airway was performed by the first author (K. Okuno) blinded to the PSG data. Image analysis software (ImageJ; http://imagej.nih.gov/ij/) was used to determine cross-sectional areas (CSA) as well as the lateral and anteroposterior (AP) diameters for both the centric occlusal position and the maximum comfortable protrusion position of the mandible. The expansion ratio was determined for the CSA, and lateral and AP diameters. The expansion ratio was calculated by dividing the measurements in the protruded position by the measurement in the centric occlusion position (figure 1). For the measurements of the oro/hypopharynx, we have noted that with mandibular advancement the epiglottis assumed a higher position, becoming closer to the tip of the fibrescope. To overcome this change in the epiglottis position, we further calibrated the images according to the lateral width of the epiglottis. For this calibration the CSA of the oro/hypopharynx was divided by the square of lateral width of the epiglottis. The lateral and AP diameters of the oro/hypopharynx were divided by the lateral width of the epiglottis. To assess the interobserver repeatability of the expansion ratio, all images were measured independently by two authors (K. Okuno and Y. Sasao) and the intra-class correlation coefficient value was calculated between the two sessions.

\section{Oral appliance}

An acrylic monobloc mandibular advancement appliance (ERKODENT, Pfalzgrafenweiler, Germany) was custom-made for each patient. Initially, mandible advancement was set at $60-70 \%$ of the maximum mandibular protruded position. The appliance was incrementally titrated to either a maximal comfortable protruded position of the mandible or a resolution of snoring and daytime symptoms. Increased advancement of the appliance was facilitated by the separation of the upper and lower components of the appliance, and then repositioning at a more advanced mandibular position.

\section{Assessment of treatment outcome}

Following endoscopic evaluation of the upper airway, each patient was provided with a custom-fitted oral appliance and, as per standard clinical procedure, underwent an adaptation and titration period over 2 months. Treatment efficacy was determined by a follow-up PSG, which was performed with the titrated oral appliance in place.

Treatment success was defined by three common criteria for the reduction in AHI. Responders according to criterion 1 were defined as patients with a reduction in AHI to $<5$ events $\mathrm{h}^{-1}$ in addition to a $>50 \%$ reduction in baseline AHI. Responders according to criterion 2 were defined as patients with a reduction in $\mathrm{AHI}$ to $<10$ events $\mathrm{h}^{-1}$ in addition to a $>50 \%$ reduction in baseline AHI. Responders according to criterion 3 were defined as patients with a $>50 \%$ reduction in baseline AHI.

\section{Statistical analysis}

All data were analysed by SPSS 15.0 statistical software (SPSS, Chicago, IL, USA). Descriptive statistics for clinical characteristics are presented as mean $\pm \mathrm{SD}$. The CSA, lateral and AP expansion ratios are presented 
a) Velopharynx
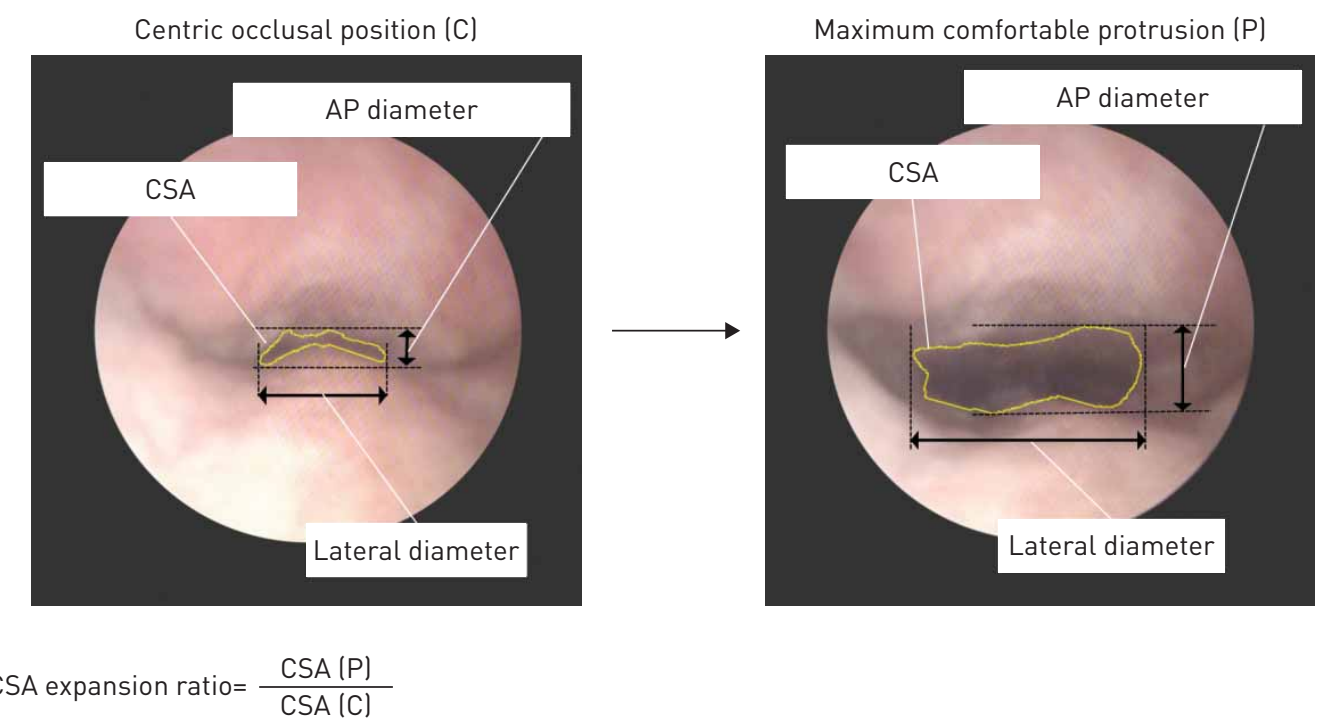

CSA expansion ratio $=\frac{\mathrm{CSA}(\mathrm{P})}{\mathrm{CSA}(\mathrm{C})}$

Lateral and AP expansion ratios are calculated by the same method

b) Oro/hypopharynx
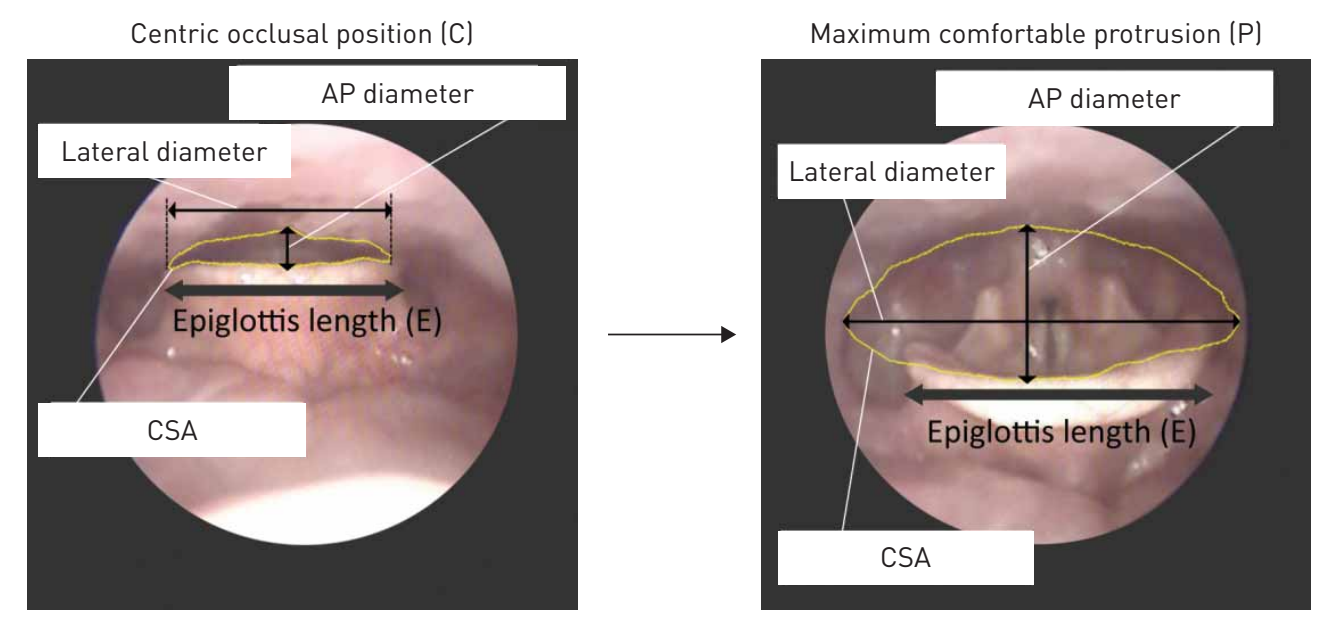

CSA expansion ratio $=\frac{\operatorname{CSA}(P) /(E(P))^{2}}{\operatorname{CSA}(C) /(E(C))^{2}}$

Lateral expansion ratio $=\frac{\text { Lateral diameter }(\mathrm{P}) / \mathrm{E}(\mathrm{P})}{\text { Lateral diameter }(\mathrm{C}) / \mathrm{E}(\mathrm{C})}$

AP expansion ratio is calculated by the same method

FIGURE 1 Upper airway evaluation using endoscopic findings. The outline of the approximate cross-sectional area (CSA), lateral diameter and anteroposterior (AP) diameter were calculated using Image $J$ software. The CSA, lateral and AP expansion ratios at the a) velopharynx and b) oro/hypopharynx were calculated by dividing the measurement in the maximum comfortable protrusion position by the measurement in the centric occlusal position. The images of the oro/hypopharynx in b) are further calibrated according to the lateral length of the epiglottis (E).

as median (interquartile range). The normality of the data distribution was assessed using the Kolmogorov-Smirnov test. An unpaired t-test or Mann-Whitney U-test was used to compare the PSG variables between responders and nonresponders, as appropriate. Multivariable logistic regression analysis was performed with oral appliance treatment outcome based on criterion 1, criterion 2 or criterion 3 as the dependent variable, and independent variables including age, body mass index (BMI), baseline AHI, and expansion ratio of CSA in the velopharynx and oro/hypopharynx. A stepwise forward selection 
procedure was also performed to examine the effects of different variables and identify the important explanatory variables. A receiver operator characteristic (ROC) curve analysis was used to determine the prediction and the best cut-off value for the expansion ratio. A $\mathrm{p}$-value of $<0.05$ was considered to indicate statistical significance.

\section{Results}

All 61 recruited patients, including 25 moderate OSA patients and 36 severe OSA patients, completed the trial. The majority $(82 \%)$ of patients were male, with a mean age of 56 years and patients were mostly overweight by Japanese standards, as seen in table 1. Patients were divided into responders and nonresponders according to criterion 1, criterion 2 and criterion 3. Baseline characteristics in the three groups are summarised in table 2 . The baseline AHI was significantly lower in responders compared with nonresponders according to criterion 1 and criterion 2 for response $(\mathrm{p}<0.05)$. Independent of the criteria, there was no significant difference between responders and nonresponders in age, BMI, baseline Epworth sleepiness scale (ESS), follow-up ESS, amount of mandibular advancement of the oral appliance or maximum mandibular protrusion.

Figure 2 presents the data for median (interquartile range) expansion ratios for the CSA, and lateral and AP diameters of the airway at the level of the velopharynx according to the three different criteria for treatment success. For all criteria, the CSA expansion ratio of the velopharynx was greater in responders compared with nonresponders (criterion 1: 2.9 (2.5-4.3) versus 1.9 (1.6-2.8), $\mathrm{p}<0.05$; criterion 2: 2.9 (2.3-5.0) versus 1.7 (1.5-1.9), $\mathrm{p}<0.001$; criterion 3: 2.5 (1.9-4.3) versus 1.8 (1.3-2.2)). To identify which dimension contributed more to predicting the success of oral appliance treatment, we compared the lateral and the AP diameter expansion ratios in the velopharynx between responders and nonresponders. For criterion 1 and criterion 2, the AP expansion ratio was greater in responders compared with nonresponders (criterion 1 , $\mathrm{p}<0.05$; criterion $2, \mathrm{p}<0.01$ ), whereas the lateral expansion ratio was greater in responders compared with nonresponders for criterion 2 and criterion 3 (criterion 2, p<0.001; criterion 3, $\mathrm{p}<0.05$ ).

Figure 3 presents the CSA, lateral and AP expansion ratios at the level of the oro/hypopharynx for the three different success criteria. The expansion ratio of the oro/hypopharynx CSA was significantly greater in responders $(3.4(2.5-5.6))$ compared with nonresponders $(2.4(1.8-3.7))(\mathrm{p}<0.05)$ for criterion 2 only. Regarding the AP and lateral dimensions, for criterion 2 there was a significant difference between responders and nonresponders in the AP expansion ratio $(\mathrm{p}<0.01)$.

The interobserver repeatability of expansion ratio measurements was determined using intra-class correlation coefficient and was found to be high and acceptable (at velopharynx: CSA 0.943, lateral 0.968, AP 0.921; at oro/hypopharynx: CSA 0.972, lateral 0.936, AP 0.970).

Multivariable logistic regression analysis was used to develop a predictive equation for oral appliance success. Nasoendoscopic parameters (the expansion ratio of the CSA at the velopharynx and oro/ hypopharynx), age, BMI and baseline AHI were included in the logistic regression analysis. The expansion ratio of the velopharyngeal CSA was found to be an independent predictor of oral appliance treatment outcome on all criteria (table 3).

TABLE 2 Patient characteristics of responders and nonresponders

\begin{tabular}{|c|c|c|c|c|c|c|}
\hline & \multicolumn{2}{|c|}{ Criterion 1} & \multicolumn{2}{|c|}{ Criterion 2} & \multicolumn{2}{|c|}{ Criterion 3} \\
\hline Subjects & 17 & 44 & 35 & 26 & 49 & 12 \\
\hline Male & 76.5 & 84.1 & 82.9 & 80.1 & 79.6 & 91.7 \\
\hline Age years & $53.2 \pm 11.0$ & $57.0 \pm 10.8$ & $54.0 \pm 11.1$ & $58.6 \pm 10.3$ & $55.7 \pm 10.6$ & $57.3 \pm 12.5$ \\
\hline BMI $\mathrm{kg} \cdot \mathrm{m}^{-2}$ & $25.2 \pm 3.2$ & $26.6 \pm 3.8$ & $25.5 \pm 3.3$ & $27.2 \pm 4.0$ & $26.1 \pm 3.8$ & $26.8 \pm 3.2$ \\
\hline AHI baseline events $h^{-1}$ & $29.0 \pm 12.2^{*}$ & $38.5 \pm 18.0$ & $32.2 \pm 14.0 *$ & $40.7 \pm 19.7$ & $36.8 \pm 17.6$ & $31.9 \pm 14.4$ \\
\hline ESS follow-up & $5.6 \pm 4.0$ & $5.1 \pm 3.4$ & $5.8 \pm 3.6$ & $4.6 \pm 3.4$ & $5.5 \pm 3.5$ & $4.5 \pm 3.7$ \\
\hline $\begin{array}{l}\text { Mandible advancement of oral } \\
\text { appliance } \mathrm{mm}\end{array}$ & $7.4 \pm 0.8$ & $7.6 \pm 1.5$ & $7.5 \pm 1.2$ & $7.6 \pm 1.4$ & $7.6 \pm 1.4$ & $7.4 \pm 1.1$ \\
\hline Maximum mandible advancement $\mathrm{mm}$ & $10.7 \pm 1.3$ & $10.5 \pm 1.9$ & $10.7 \pm 1.3$ & $10.3 \pm 2.2$ & $10.7 \pm 1.8$ & $9.8 \pm 1.3$ \\
\hline
\end{tabular}

Data are presented as $\mathrm{n}, \%$ or mean \pm SD. Criterion 1: reduction in apnoea-hypopnoea index (AHI) to $<5$ events. $\mathrm{h}^{-1}$ and $>50 \%$ reduction from baseline; criterion 2: reduction in $\mathrm{AHI}$ to $<10$ events $\cdot \mathrm{h}^{-1}$ and $>50 \%$ reduction from baseline; criterion 3 : reduction in $\mathrm{AHI}>50 \%$. BMI: body mass index; ESS: Epworth sleepiness scale. ${ }^{*}$ : $p<0.05$ versus nonresponders; ${ }^{* *}$ : $p<0.01$ versus nonresponders. 

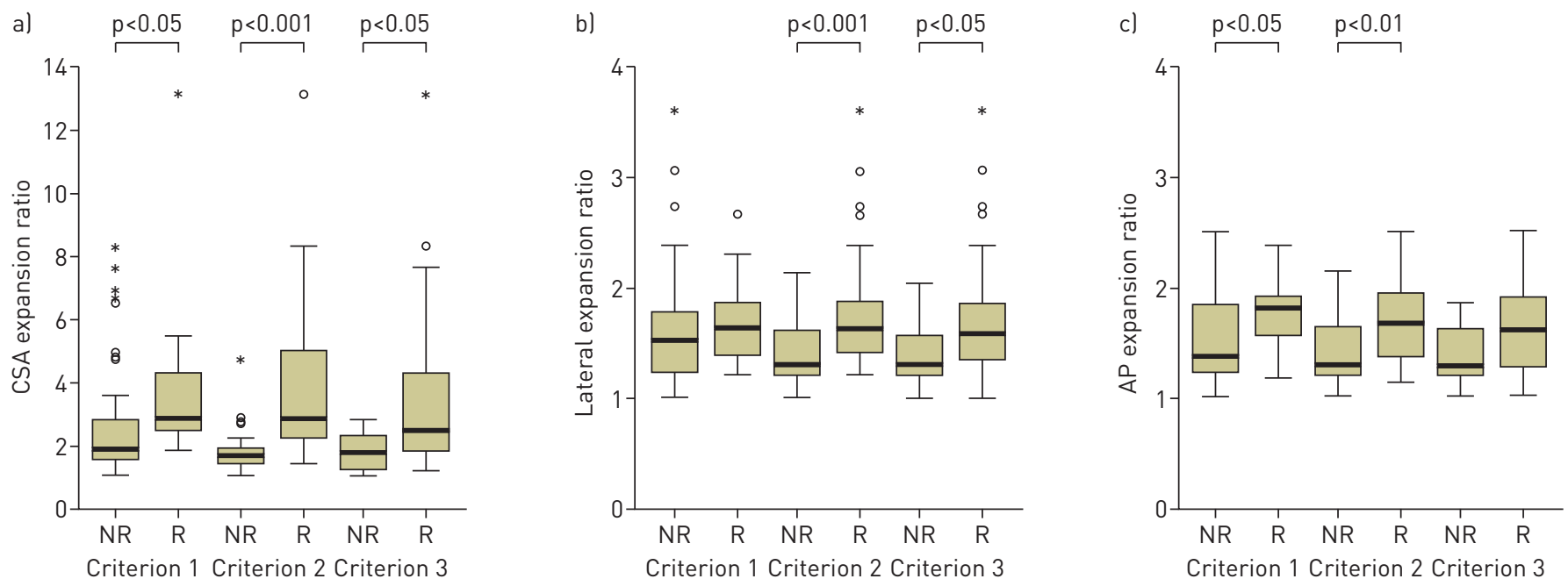

FIGURE 2 a) Cross-sectional area (CSA), b) lateral and c) anteroposterior (AP) expansion ratios at the velopharynx in responders (R) and nonresponders (NR). The expansion ratios were calculated as shown in figure 1. For all three criteria, the velopharynx CSA and AP expansion ratios were significantly greater in responders versus nonresponders. Box-and-whisker plots: median values represented by horizontal line with the box, interquartile range by box height, and minimum and maximum values within the range by whiskers. Open circles represent outlier cases (values 1.5-3 box lengths above box edges); asterisks represent extreme cases (values $>3$ box lengths above box edges). Criterion 1: reduction in apnoea-hypopnoea index (AHI) to $<5$ events $\cdot \mathrm{h}^{-1}$ and $>50 \%$ reduction from baseline; criterion 2: reduction in $\mathrm{AHI}$ to $<10$ events $\cdot \mathrm{h}^{-1}$ and $>50 \%$ reduction from baseline; criterion 3: reduction in $\mathrm{AHI}>50 \%$.
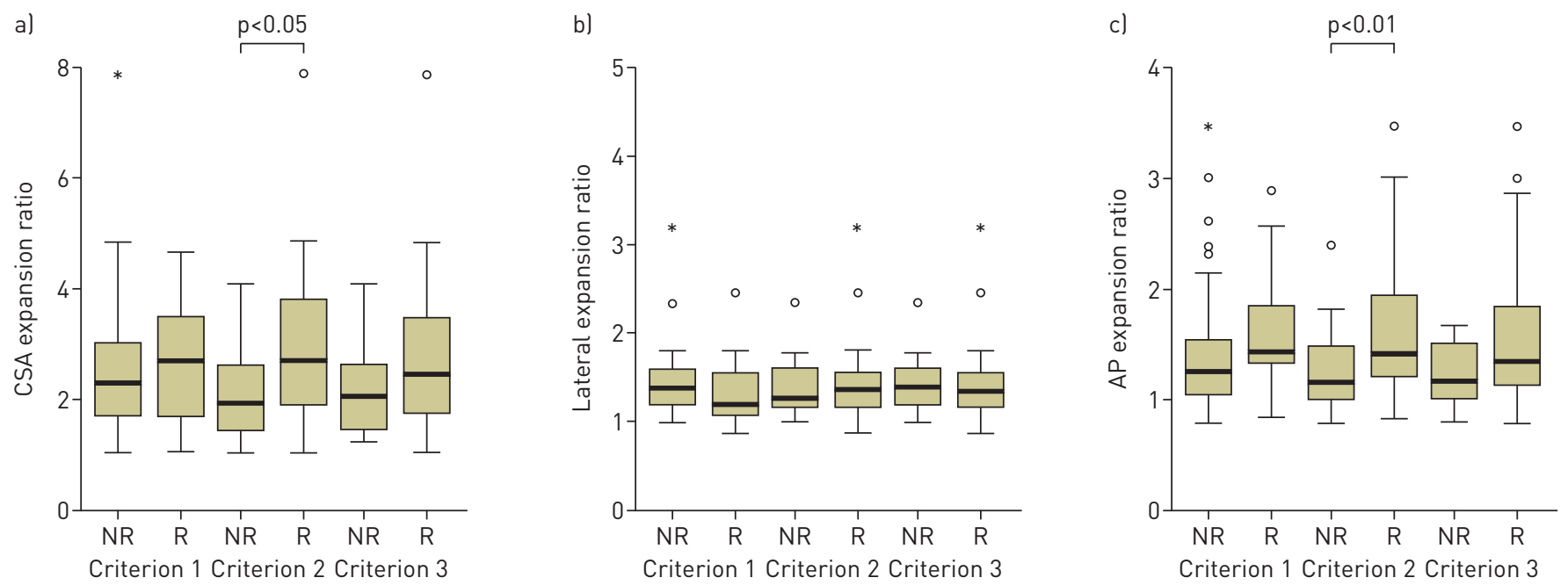

FIGURE 3 a) Cross-sectional area (CSA), b) lateral and c) anteroposterior (AP) expansion ratio at the oro/hypopharynx in responders (R) and nonresponders (NR). The expansion ratios were calculated as shown in figure 1. The oro/hypopharynx CSA expansion ratio was significantly greater in responders versus in nonresponders only for criterion 2. Box-and-whisker plots: median values represented by horizontal line with the box, interquartile range by box height, and minimum and maximum values within the range by whiskers. Open circles represent outlier cases (values 1.5-3 box lengths above box edges) and the asterisks represent extreme cases (values $>3$ box lengths above box edges). Criterion 1: reduction in apnoea-hypopnoea index (AHI) to $<5$ events $\cdot \mathrm{h}^{-1}$ and $>50 \%$ reduction from baseline; criterion 2 : reduction in $\mathrm{AHI}$ to $<10$ events $\cdot \mathrm{h}^{-1}$ and $>50 \%$ reduction from baseline; criterion 3 : reduction in $\mathrm{AHI}>50 \%$ 


\begin{tabular}{|c|c|c|c|c|c|}
\hline Variable & $\boldsymbol{\beta}$ & SE & Wald & OR & p-value \\
\hline \multicolumn{6}{|l|}{ Criterion 1} \\
\hline AHI baseline events $\cdot \mathrm{h}^{-1}$ & -0.61 & 0.029 & 4.517 & 0.941 & 0.034 \\
\hline Expansion ratio of CSA in the velopharynx & 0.349 & 0.166 & 4.434 & 1.418 & 0.035 \\
\hline \multicolumn{6}{|l|}{ Criterion 2} \\
\hline AHI baseline events $\cdot \mathrm{h}^{-1}$ & -0.087 & 0.030 & 8.229 & 0.916 & 0.004 \\
\hline Expansion ratio of CSA in the velopharynx & 1.992 & 0.604 & 10.864 & 7.328 & 0.001 \\
\hline \multicolumn{6}{|l|}{ Criterion 3} \\
\hline Expansion ratio of CSA in the velopharynx & 1.018 & 0.505 & 4.060 & 2.766 & 0.044 \\
\hline
\end{tabular}

Criterion 1: reduction in apnoea-hypopnoea index (AHI) to $<5$ events $\cdot \mathrm{h}^{-1}$ and $>50 \%$ reduction from baseline; criterion 2: reduction in $\mathrm{AHI}$ to $<10$ events $\cdot \mathrm{h}^{-1}$ and $>50 \%$ reduction from baseline; criterion 3: reduction in $\mathrm{AHI}>50 \%$. CSA: cross-sectional area.

\section{Discussion}

This study demonstrates that, prior to the initiation of treatment, nasoendoscopy is a useful tool in the prediction of oral appliance treatment outcome. This predictive method is clinically straightforward, and has good sensitivity and specificity. The results of this study are the first to demonstrate the clinical utility of nasoendoscopy for the prediction of oral appliance treatment outcome in patients with moderate and severe OSA using quantitative and prospective methodology.

In the evaluation of the upper airway, cephalometry and computed tomography (CT) as well as magnetic resonance imaging (MRI) have been used to predict the severity of OSA and effects of treatment. Lateral cephalometry is often used in clinical practice to identify craniofacial characteristics in patients with OSA because it is inexpensive, widely available and associated with minimal radiation exposure [21]. However, cephalometry is limited to two dimensions in the mid-sagittal plane and is unable to evaluate the airway in the lateral dimension. CT is a very useful method to evaluate the upper airway, and it has been reported that a combination of the smallest CSA using CT and BMI are highly predictive of OSA severity [22]. MRI of the upper airway has previously been used to demonstrate that an improvement in upper airway patency with mandibular advancement was associated with a successful treatment outcome [23]. However, these techniques are difficult to use in a clinical practice due to high costs and/or a high radiation exposure dose (CT). In contrast, nasoendoscopy allows the evaluation of changes in the upper airway in both the lateral and AP dimensions [20, 24, 25], and is widely available and easily performed in clinical practice. With regard to quantification accuracy, it has been reported that the agreement between the video endoscopic image and MRI measurements was $93.3 \%$ at the retropalatal level and $95.6 \%$ at the retrolingual level of the pharynx [26]. Interobserver agreement in measurement is also high, as seen in our study.
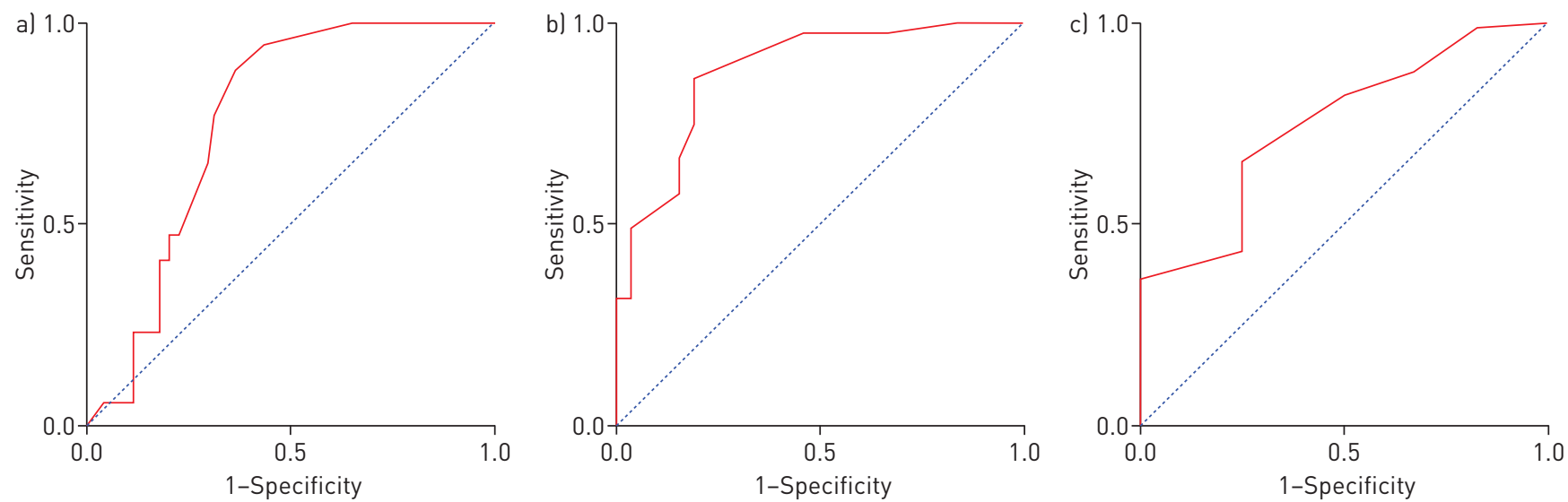

FIGURE 4 Receiver operating characteristic curves for the expansion ratio of the cross-sectional area in the velopharynx. a) Criterion 1: reduction in apnoea-hypopnoea index (AHI) to $<5$ events $\cdot \mathrm{h}^{-1}$ and $>50 \%$ reduction from baseline (best cut-off value 2.35 , sensitivity $88.2 \%$, specificity $68.2 \%$, positive predictive value (PPV) $51.7 \%$, negative predictive value (NPV) $93.8 \%$, area under curve (AUC) 0.76 ). b) Criterion 2: reduction in AHI to $<10$ events $\cdot h^{-1}$ and $>50 \%$ reduction from baseline (best cut-off value 2.00 , sensitivity $85.7 \%$, specificity $80.8 \%$, PPV $85.7 \%$, NPV $80.8 \%$, AUC 0.87 )

c) Criterion 3: reduction in $\mathrm{AHI}>50 \%$ (best cut-off value 2.00, sensitivity $65.3 \%$, specificity $75.0 \%, \mathrm{PPV} 91.4 \%, \mathrm{NPV} 34.6 \%, \mathrm{AUC} 0.74$ ). 
TABLE 4 Prediction of oral appliance treatment response by nasoendocsopic measurement of the cross-sectional area in the velopharynx

\begin{tabular}{lccc} 
& Criterion 1 & Criterion 2 & Criterion 3 \\
\hline AUC & $0.76(0.65-0.88)$ & $0.87(0.78-0.96)$ & $0.74(0.59-0.89)$ \\
Best cut-off expansion ratio & 2.35 & 2.00 & 2.00 \\
Sensitivity & 88.2 & 85.7 & 65.3 \\
Specificity & 68.2 & 80.8 & 75.0 \\
PPV & 51.7 & 85.7 & 91.4 \\
NPV & 93.8 & 80.8 & 34.6 \\
\hline
\end{tabular}

Data are presented as mean $(95 \% \mathrm{CI}), \mathrm{n}$ or $\%$. Criterion 1: reduction in apnoea-hypopnoea index (AHI) to $<5$ events $\cdot \mathrm{h}^{-1}$ and $>50 \%$ reduction from baseline; criterion 2: reduction in AHI to $<10$ events $\cdot \mathrm{h}^{-1}$ and $>50 \%$ reduction from baseline; criterion 3: reduction in $\mathrm{AHI}>50 \%$. AUC: area under the curve; PPV: positive predictive value; NPV: negative predictive value.

Given these considerations, we feel that the described nasoendoscopic approach has the potential to be a useful tool for prediction of oral appliance treatment outcome.

Recently, we reported that the effects of oral appliance therapy could be predicted by the presence of velopharyngeal widening and suggested a nasoendoscopic approach to the prediction of oral appliance treatment outcomes [16]. VRoEgor et al. [15] showed a statistically significant association between a positive effect of the simulation bite on upper airway patency during DISE and treatment response with oral appliance treatment. However, the study had several limitations in that assessment of the upper airway during DISE was based on subjective findings and the DISE approach is difficult to use in routine clinical practice due to the required administration of propofol in a controlled setting under the supervision of specialised healthcare professionals. CHAN et al. [17] demonstrated that an increase in the CSA of the airway lumen when the Muller manoeuvre was performed during mandibular advancement was associated with a PSG treatment response for the velopharyngeal and hypopharyngeal segments. Although assessment of the airway lumen was a quantitative evaluation in that study, the study design was retrospective because the endoscopic assessment was undergone after a period of adaptation and usage of an oral appliance, which could be related to a decrease in upper airway oedema. The authors suggested the importance of a prospective study of nasopharynx evaluation prior to construction of the oral appliance.

A number of studies have reported the importance of the evaluation of the upper airway at the level of the velopharynx and oro/hypopharynx [27]. Therefore, we calculated the expansion ratio of CSA at the level of the velopharynx and oro/hypopharynx during maximal comfortable protrusion. Using three different criteria for success, the expansion ratio of the velopharynx CSA was significantly greater in responders than in nonresponders. In contrast, the expansion ratio of the oro/hypopharynx CSA did not show consistent results using either criterion, although it was significant for criterion 2. Multivariable logistic regression analysis revealed that the expansion ratio of the velopharyngeal CSA was a significant independent predictor of oral appliance treatment outcome based on all criteria. These results suggest that the widening of the velopharynx with mandibular advancement may be more important than the widening of the oro/ hypopharynx for the prediction of oral appliance treatment outcome, similar to previous studies [15-17, 28].

Recently, a review study stated that an endoscopic assessment of the upper airway in OSA patients should include the level, degree and direction of obstruction [27]. We have previously hypothesised on the importance of the direction of upper airway widening on the AHI reduction rate associated with oral appliance treatment [16]. In the present study, we evaluated the expansion ratio of not only the CSA but also the lateral and AP dimensions at the level of the velopharynx and oro/hypopharynx. At the level of the velopharynx, the AP expansion ratio was significantly greater in responders than in nonresponders for criterion 1 and criterion 2. With regard to dimension, our results suggest that AP velopharyngeal widening may be an important predictor of oral appliance treatment success.

In order to predict oral appliance treatment outcome, the best cut-off value for the expansion ratio of the velopharyngeal CSA was $\sim 2.0$ for the three different success criteria. This value of the expansion ratio corresponds to a doubling in size of the velopharyngeal lumen with mandibular protrusion for oral appliance responders. The AUC of the expansion ratio in the velopharynx for prediction of success ranged from 0.74 to 0.87 , indicating good model discrimination. For the more rigorous success criterion 1 , it was found to be a good predictor of a poor response to oral appliance therapy with a high NPV (93.8\%). For the less stringent success criterion 2, the expansion ratio of the velopharynx was able to predict a successful treatment outcome with high accuracy (sensitivity $85.7 \%$, specificity $80.8 \%$, PPV $85.7 \%$, NPV 
80.8\%). Hence, we believe it has the potential to become a useful prediction tool in clinical practice. Multivariable logistic regression analysis revealed that the expansion ratio of the velopharyngeal CSA was a significant independent predictor of oral appliance treatment outcome on three different success criteria, and baseline AHI was a significant independent predictor for criterion 1 and 2 . In agreement with our current study, it is well recognised that lower baseline AHI is associated with a better response to oral appliance treatment [9].

As the success rates of oral appliances are usually lower with increasing OSA severity (e.g. 54\% failure in severe OSA versus 30\% failure in mild OSA [29]), it is highly important to predict treatment efficacy, especially in patients with moderate-to-severe OSA. Therefore, the current study included patients with moderate-to-severe OSA.

The titration protocol used in the present study was based on a combination of symptomatic improvement and maximum comfortable mandibular protrusion. Although there are many titration protocols for oral appliances, including titration procedures combining the analysis of clinical improvement and limited sleep study data, our protocol is widely accepted and used in clinical practice. While it is possible that titration was suboptimal in some patients due to a lack of objective data, a recent review presenting an overview of various titration methods, including subjective, objective and combinations of both protocols, concluded that at the present time there is no gold standard method for titration protocols [30].

This study has a number of potential limitations. As all study subjects presented to the dental hospital clinic with an expressed interested in oral appliance therapy, there was the potential for selection bias. However, we consider this to be of minimal influence as the number of nonresponders (criterion 1: $72 \%$; criterion 2: 43\%; criterion 3: 20\%) was similar to those in previous studies. Another potential limitation is the limited sample size and the relatively lower BMI than other studies. The World Health Organization Expert Consultation has reported that for many Asian populations trigger points for public health action were identified as $\geqslant 23 \mathrm{~kg} \cdot \mathrm{m}^{-2}$, because Asians generally have a higher percentage of body fat than Caucasians of the same BMI [31]. Also, the Committee of Japan Society for the Study of Obesity reported that the criteria for obesity disease for Japanese was defined as a BMI $\geqslant 25 \mathrm{~kg} \cdot \mathrm{m}^{-2}$ [32]. Therefore, our Japanese sample (mean BMI $26.2 \mathrm{~kg} \cdot \mathrm{m}^{-2}$ ) was considered obese for their standard. To better understand if this study is valid in a Caucasian obese population, further trials with the same methodology are still required. Also, the amount of mandibular advancement during nasoendoscopy was not specifically quantified, rather the maximum comfortable protrusion for the patient was used, as in a previous study [16]. This may be a limitation of the current protocol, but the main reason of this approach was to develop a clinically simple useful method for predicting treatment success. Therefore, we opted for using a practical protocol for mandibular protrusion, which is more likely to be used and implemented outside of a research setting. JOHAL et al. [12, 13] and BATTAGEL et al. [14] have also reported that mimicking the action of a mandibular advancement device during nasoendoscopy helps to predict successful oral appliance therapy. A limitation of this study is the lack of vertical opening assessment in performing nasoendoscopy. Patients were asked to protrude their mandible while keeping their teeth in contact. With this procedure, there was no space for an oral appliance and increased vertical opening is a factor known to worsen OSA. Despite this limitation, the prediction of treatment outcome with our current method was very good and it may be related to the oral appliance used, which had a minimal vertical increase.

The study protocol was also performed with the subjects awake. The degree of upper airway narrowing has been shown to increase according to sedation depth [33], suggesting different airway dimensions between the sleep and awake states. DISE has been shown to be a useful diagnostic tool for the upper airway in snoring and OSA because the sites and patterns of upper airway collapse during sleep can be significantly different to those observed in awake patients, primarily due to differences in muscle tone [27]. It has been reported that DISE assists in the surgical planning for sleep-disordered breathing [34]. However, with regard to the prediction of oral appliance treatment outcomes, DISE is not generalisable and not accessible in routine clinical practice [15]. Despite these limitations, this study strongly suggests that velopharyngeal widening as visualised by nasoendoscopy can predict the outcome of oral appliance treatment in OSA subjects.

In conclusion, the CSA expansion ratio of the velopharynx was significantly greater in responders compared to nonresponders and a high level of predictive accuracy was found for a cut-off value of 2.0. Based on these results, nasoendoscopy may have significant clinical utility to predict success of oral appliance treatment. 


\section{References}

1 Young T, Palta M, Dempsey J, et al. The occurrence of sleep-disordered breathing among middle-aged adults. $N$ Engl J Med 1993; 328: 1230-1235.

2 Flemons WW. Obstructive sleep apnea. N Engl J Med 2002; 347: 498-504.

3 Kribbs NB, Pack AI, Kline LR, et al. Objective measurement of patterns of nasal CPAP use by patients with obstructive sleep apnea. Am Rev Respir Dis 1993; 147: 887-895.

4 McArdle N, Devereux G, Heidarnejad H, et al. Long-term use of CPAP therapy for sleep apnea/hypopnea syndrome. Am J Respir Crit Care Med 1999; 159: 1108-1114.

5 Weaver TE, Kribbs NB, Pack AI, et al. Night-tonight variability in CPAP use over the first three months of treatment. Sleep 1997; 20: 278-283.

6 Gagnadoux F, Fleury B, Vielle B, et al. Titrated mandibular advancement versus positive airway pressure for sleep apnoea. Eur Respir J 2009; 34: 914-920.

7 Sutherland K, Vanderveken OM, Tsuda H, et al. Oral appliance treatment for obstructive sleep apnea: an update. J Clin Sleep Med 2014; 10: 215-227.

8 Ramar K, Dort LC, Katz SG, et al. Clinical practice guideline for the treatment of obstructive sleep apnea and snoring with oral appliance therapy: an update for 2015. J Clin Sleep Med 2015; 11: 773-827.

9 Ferguson KA, Cartwright R, Rogers R, et al. Oral appliances for snoring and obstructive sleep apnea: a review. Sleep 2006; 29: 244-262.

10 Pelletier-Fleury N, Meslier N, Gagnadoux F, et al. Economic arguments for the immediate management of moderate-to-severe obstructive sleep apnoea syndrome. Eur Respir J 2004; 23: 53-60.

11 Hessel NS, de Vries N. Results of uvulopalatopharyngoplasty after diagnostic workup with polysomnography and sleep endoscopy: a report of 136 snoring patients. Eur Arch Otorhinolaryngol 2003; 260: 91-95.

12 Johal A, Hector MP, Battagel JM, et al. Impact of sleep nasendoscopy on the outcome of mandibular advancement splint therapy in subjects with sleep-related breathing disorders. J Laryngol Otol 2007; 121: 668-675.

13 Johal A, Battagel JM, Kotecha BT. Sleep nasendoscopy: a diagnostic tool for predicting treatment success with mandibular advancement splints in obstructive sleep apnoea. Eur J Orthod 2005; 27: 607-614.

14 Battagel JM, Johal A, Kotecha BT. Sleep nasendoscopy as a predictor of treatment success in snorers using mandibular advancement splints. J Laryngol Otol 2005; 119: 106-112.

15 Vroegop AV, Vanderveken OM, Dieltjens M, et al. Sleep endoscopy with simulation bite for prediction of oral appliance treatment outcome. J Sleep Res 2013; 22: 348-355.

16 Sasao Y, Nohara K, Okuno K, et al. Videoendoscopic diagnosis for predicting the response to oral appliance therapy in severe obstructive sleep apnea. Sleep Breath 2014; 18: 809-815.

17 Chan AS, Lee RW, Srinivasan VK, et al. Nasopharyngoscopic evaluation of oral appliance therapy for obstructive sleep apnoea. Eur Respir J 2010; 35: 836-842.

18 Iber C, Ancoli-Israel S, Chesson AL, et al. The AASM Manual for the Scoring of Sleep Associated Events: Rules, Terminology and Technical Specifications. 1st Edn. Westchester, American Academy of Sleep Medicine, 2007.

19 Rechtschaffen A, Kales A. A Manual of Standardized Terminology, Techniques and Scoring System for Sleep Stages of Human Subjects. Los Angeles, Brain Information Service/Brain Research Institute, University of California, 1968.

20 Ryan CF, Love LL. Mechanical properties of the velopharynx in obese patients with obstructive sleep apnea. Am J Respir Crit Care Med 1996; 154: 806-812.

21 Togeiro SM, Chaves CM Jr, Palombini L, et al. Evaluation of the upper airway in obstructive sleep apnoea. Indian I Med Res 2010; 131: 230-235.

22 Vos W, De Backer J, Devolder A, et al. Correlation between severity of sleep apnea and upper airway morphology based on advanced anatomical and functional imaging. J Biomech 2007; 40: 2207-2213.

23 Sanner BM, Heise M, Knoben B, et al. MRI of the pharynx and treatment efficacy of a mandibular advancement device in obstructive sleep apnoea syndrome. Eur Respir J 2002; 20: 143-150.

24 Ferguson KA, Love LL, Ryan CF. Effect of mandibular and tongue protrusion on upper airway size during wakefulness. Am J Respir Crit Care Med 1997; 155: 1748-1754.

25 Ryan CF, Love LL, Peat D, et al. Mandibular advancement oral appliance therapy for obstructive sleep apnoea: effect on awake calibre of the velopharynx. Thorax 1999; 54: 972-977.

26 Hsu PP, Han HN, Chan YH, et al. Quantitative computer-assisted digital-imaging upper airway analysis for obstructive sleep apnoea. Clin Otolaryngol Allied Sci 2004; 29: 522-529.

27 De Vito A, Carrasco Llatas M, Vanni A, et al. European position paper on drug-induced sedation endoscopy (DISE). Sleep Breath 2014; 18: 453-465.

28 Isono S, Tanaka A, Sho Y, et al. Advancement of the mandible improves velopharyngeal airway patency. $J$ Appl Physiol 1995; 79: 2132-2138.

29 Phillips CL, Grunstein RR, Darendeliler MA, et al. Health outcomes of continuous positive airway pressure versus oral appliance treatment for obstructive sleep apnea: a randomized controlled trial. Am J Respir Crit Care Med 2013; 187: 879-887.

30 Dieltjens M, Vanderveken OM, Heyning PH, et al. Current opinions and clinical practice in the titration of oral appliances in the treatment of sleep-disordered breathing. Sleep Med Rev 2012; 16: 177-185.

31 WHO Expert Consultation. Appropriate body-mass index for Asian populations and its implications for policy and intervention strategies. Lancet 2004; 363: 157-163.

32 Takahashi H, Mori M. [Characteristics and significance of criteria for obesity disease in Japan 2011]. Nihon Rinsho 2013; 71: 257-261.

33 Hong SD, Dhong HJ, Kim HY, et al. Change of obstruction level during drug-induced sleep endoscopy according to sedation depth in obstructive sleep apnea. Laryngoscope 2013; 123: 2896-2899.

34 Gillespie MB, Reddy RP, White DR, et al. A trial of drug-induced sleep endoscopy in the surgical management of sleep-disordered breathing. Laryngoscope 2013; 123: 277-282. 